\title{
The eGFR Change and Risk Factors in Moderate Chronic Kidney Disease Patients after Successful HCV Clearance by Direct Anti-viral Agents
}

Yen-Chi Hu

Taipei Veterans General Hospital

Keng-Hsin Lan ( $\square$ khlan@vghtpe.gov.tw )

Taipei Veterans General Hospital

Chia-Ling Lu

Taipei Veterans General Hospital

Yi-Hsiang Huang

Taipei Veterans General Hospital

Ming-Chih Hou

Taipei Veterans General Hospital

\section{Research Article}

Keywords: HCV, Direct Anti-viral Agent, Chronic kidney disease

Posted Date: May 18th, 2021

DOl: https://doi.org/10.21203/rs.3.rs-514699/v1

License: (9) This work is licensed under a Creative Commons Attribution 4.0 International License.

Read Full License 
Title: The eGFR change and risk factors in moderate Chronic kidney disease patients after successful HCV clearance by Direct anti-viral agents Yen-Chi Hu 1, Chia-ling Lu 1, Yi-Hsiang Huang 1, Ming-Chih Hou 1, Keng-Hsin Lan 1 (corresponding author)

1. Taipei Veterans General Hospital The Division of Gastroenterology \& Hepatology 
Abstract:

Background -Chronic HCV infection is related not only to chronic kidney disease (CKD) but also accelerates renal deterioration. Treatment with Direct-acting antiviral agents (DAA) could slow renal function decline in some trials, but the long-term outcomes of renal function changes following HCV elimination by DAA remained inconclusive.

Methods - This retrospective study analyzed the data of HCV infected patients with CKD stage 3 who were treated with DAA and achieved sustained virologic response at 12weeks after treatment (SVR12) during 2017-2020 at a single medical center. Results - Among $130 \mathrm{HCV}$ infection and CKD stage 3 patients treated with DAA, 77 patients had no eGFR decline at SVR 12, and 53 patients had eGFR declined at SVR 12. The eGFR change on SVR 12 can be predictor for eGFR change on SVR96 (Odd ratio 3.088, $p=0.053)$. Patients with Diabetes Mellites $(D M)(p=0.016, O R 2.6)$ is highly associated with eGFR decline after DAA treatment. Renal function deterioration during DAA treatment is associated to long-term renal function decline $(p=0.000)$. Lower HCV RNA titer $(p=0.024)$, higher baseline MELD score $(p=0.008)$, or con-current malignant disease under treatment $(p=0.044)$ are more vulnerable to eGFR decrease upon DAA treatment.

Conclusion -Among patients with HCV infection and CKD stage 3, comorbidity with 
DM, have less benefit to renal function after HCV elimination by DAA. Higher baseline HCV RNA viral load and MELD score are precipitating factors to the renal function impairment. Treatment to malignant disease, either by systemic or localized treatment, increases the risk of renal function impairment during DAA treatment. The eGFR change on SVR 12 can be used to predict long-term eGFR change for the CKD stage 3 patients. 
Key words: HCV, Direct Anti-viral Agent, Chronic kidney disease 
Introduction:

The HCV infection is a common chronic viral infection which leads to chronic kidney disease (CKD) progression and increased incidence of End stage renal disease (ESRD)[1-4]. Kidney disorder appears in $10 \%$ to $60 \%$ of patients.[5] It is documented that HCV infection injures kidneys mainly via immune mediated tissue damage and cryoglobulinemia.[6-9]. Various histological types of renal diseases have been reported in association with HCV infection, such as membranoproliferative nephropathy (MPGN), focal segmental glomerulosclerosis (FSGS), fibrillary glomerulonephritis, immunotactoid glomerulopathy, IgA nephropathy, renal thrombotic microangiopathy, renal vasculitis, and interstitial nephritis. [10]. A SVR is usually associated with a reversal of HCV related manifestations.[8]. Effects of SVR on renal function in interferon (IFN)-based treatment has been published with a national wide cohort study in Taiwan. $[11,12]$ The manifestation of proteinuria, cryoglobulinemic MPGN, FSGS, and IgA nephropathy were reported stabilization and improvement with IFN therapy. [10] The decrease in proteinuria was found to be associated with HCV-RNA clearance, and stabilization of serum creatine was achieved. [13]

The renal safety of anti-viral agents on renal function in severe CKD or ESRD had been published in several studies, and sofosbuvir-based regimens as well. However, 
discordant results on renal function after HCV clearance by DAA were reported. In an Italian cohort study for 3264 patients treated with HCV by DAA with 96\% achieved SVR [14], the eGFR was found decrease about $3 \mathrm{ml} / \mathrm{min} / 1.73 \mathrm{~m}^{2}$ in all treated patients at SVR12, while the patients with CKD3A increased their eGFR about 2 $\mathrm{ml} / \mathrm{min} / 1.73 \mathrm{~m}^{2}$ and CKD3B increased about $1 \mathrm{ml} / \mathrm{min} / 1.73 \mathrm{~m}^{2}$ at SVR12. Another cohort study for 1590 patients in Boston treated HCV with DAA and SVR12 (90.7\%) [15] had overall eGFR improvement about $3 \mathrm{ml} / \mathrm{min} / 1.73 \mathrm{~m}^{2}$. Patients with eGFR $<60$ $\mathrm{ml} / \mathrm{min} / 1.73 \mathrm{~m}^{2}$ experienced a significant stabilization of eGFR decline after DAAs while more episodes of acute kidney Injury (AKI) appeared. The kidney injury could be irreversible even after DAA discontinuation. A meta-analysis for the renal function and DAA treatment documented that patients with eGFR $<60 \mathrm{ml} / \mathrm{min} / 1.73 \mathrm{~m}^{2}$ had a higher risk to renal function deterioration, while the risk factors had not been reported. [5] Whether renal outcome could be improved in patients with moderate chronic kidney disease after HCV clearance by DAA, as well as the risk factors to renal function damage, is not well investigated. [9]

Therefore, the aims of this study were to determine the risk factors for eGFR change and its predicting factors to CKD stage 3 patients treated with DAA. 


\section{Material and Methods:}

\section{Study population}

Between 2017-2020, 1191 patients with HCV infection and received Direct Anti-

Viral Agents (DAA) at Taipei Veterans General Hospital were enrolled in this

retrospective observational cohort study. The use of DAA regimens was based on the

physician's discretion and HCV genotype. HCV infection was defined as detectable

HCV antibody (Anti-HCV; cobas-e801 analytical unit, F. Hoffmann-La Roche AG, Swiss)

and quantifiable serum HCV RNA (cobas-6800 analytical unit, F. Hoffmann-La Roche

AG, Swiss). Patients included to the study had moderate chronic kidney disease,

having estimated glomerular infiltration rate (eGFR) $30<=e G F R<60 \mathrm{ml} / \mathrm{min} / 1.73 \mathrm{~m}^{2}$

by Modification of Diet in Renal Disease (MDRD) equation, defined as CKD stage 3 by

Taiwan Chronic kidney disease clinical guidelines, 2015. The exclusion criteria were

incomplete treatment, loss follow up at SVR 12, and failure of HCV treatment. The

study was approved by VGHtpe Ethic Committee with principles of Declaration of

Helsinki and the international conference on Harmonization for good clinical practice.

Informed and consent were obtained from the enrolled patients.

\section{Study design}

Baseline patient demographics, including age, sex, history of underlying diseases 
(Diabetes mellites (DM) and hypertension (HTN)), operation history, history of organ transplantation, body mass index (BMI), and use of concomitant nephrotoxic agents were collected. Anti-HIV (cobas-e801 analytical unit, F. Hoffmann-La Roche AG, Swiss), HBV surface antigen (HBsAg) (cobas-e801 analytical unit, F. Hoffmann-La Roche AG, Swiss), anti-HCV, HCV RNA, HCV genotype (cobas-480 analytical unit, F. Hoffmann-La Roche AG, Swiss) were assessed for all patients. Severity of liver cirrhosis was estimated by fib-4 score, and the liver function was estimated by Model for End-Stage Liver Disease (MELD) score, Albumin-Bilirubin (ALBI) score, and ChildTurcotte-Pugh (CTP) score.

The hemogram, international normalized ratio, serum albumin, total/direct bilirubin, aspartate aminotransferase (AST), alanine aminotransferase (ALT), and creatinine were assessed at baseline, on-treatment weeks 4,8,12, (and 24), and off treatment weeks of $12,24,48$, and 96 . The eGFR was measured by using MDRD equation. All events or episodes related to medical issues were recorded, such as liver decompensation, infection, or status of autoimmune disease.

\section{Determine of virologic and clinical outcomes}

SVR was defined as an undetectable HCV RNA at least 12 weeks after the completion of therapy. Laboratory values were obtained before, during and after treatment as a 
part of routine care. It included the complete Blood Count/Differential Count, international normalized ration, serum albumin, total/direct bilirubin, aspartate aminotransferase (AST), alanine aminotransferase (ALT), creatinine, and alpha-fetal protein (AFP).

Baseline laboratory values were defined by the laboratory values obtained most close to the prescribing day of DAA. The 4 weeks (wks) laboratory values were defined as the laboratory values obtained around 28 days after DAA prescription. The 8 weeks (wks) laboratory value were defined as the laboratory values obtained around 56 days after DAA prescription. The 12 weeks (wks) laboratory value were defined as the laboratory values obtained around 84 days after DAA prescription. End of treatment (EOT) laboratory values were defined as laboratory values collected around 56 days in patients treated with 8 weeks course; 84 days in patients treated with 12 weeks course; 168 days in patients treated with 24 weeks course. The SVR 12 wks laboratory values were defined as laboratory values collected around 84 days after EOT day. The SVR 48wks laboratory values were collected around 12 months after EOT. The SVR 96wks data was collected around 24 months after EOT.

\section{Statistical analysis}

Statistical Program for Social Sciences (SPSS Statistics Version 23.0, IBM Corp., Armonk, New York, USA), Excel 2019(Microsoft 2019, Microsoft corporation) were 
used for all statistical analyses. The patient characteristics were shown as mean with standard error. The continuous variables were compared with nonparametric tests, Wilcoxon Signed-rank Test sign test or Mann-Whitney U test. The categorical variables were compared by $\mathrm{X}^{2}$ and Fisher's exact test. A p-value $<0.05$ was considered statistically significant. Results were expressed as Odds Ratio (OR) with 95\% confidence interval $(\mathrm{Cl})$. 


\section{Definitions}

Cirrhosis:

The presence of cirrhosis was defined by biopsy and/or a combination of clinical, laboratory and imaging criteria established a priori [16-19].

(i) evidence of stage 4 fibrosis by liver biopsy any time prior to therapy,

(ii) evidence of stage 3 fibrosis by liver biopsy any time prior to therapy with any of the following criteria:

platelet count $<140000$ per ul, presence of esophageal varices on PES, evidence of cirrhosis and/or portal hypertension and/or of ascites by imaging studies, FibroSure (or equivalent) test, vibration-controlled transient elastography or equivalent compatible with stage 4 fibrosis ( $\geq 12.5 \mathrm{kPa}$ ) or

(iii) In the absence of liver biopsy, >2 criteria:

(1) platelets count $<140000$ per ul

(2) presence of esophageal varices

(3) evidence of cirrhosis and/or portal hypertension and/or ascites by imaging studies, FibroSure or equivalent test, elastography or transient elastography (Fibroscan) result compatible with Metavir stage 4 fibrosis with stage 4 fibrosis ( $\geq 12.5 \mathrm{kPa})$, and Fib4 score $>3.5$.

Liver decompensation: 
Decompensation was defined by those who had either one of the following descriptions. [20](Taiwan National Health Insurance)

(i) International normalized ratio $>1.5$ (prothrombin time $>3$ seconds)

(ii) Total bilirubin $>2 \mathrm{mg} / \mathrm{dl}$, jaundice

(iii) Uncontrollable ascites

(iv) Hepatoencephalopathy.

Chronic kidney disease (CKD) staging:

The eGFR was estimated by using the equation of modification of diet in renal disease (MDRD). The stages of CKD were classified according to Kidney Disease Improving Global Outcomes (KDIGO) guidelines (2012) and Taiwan Chronic Kidney Disease Clinical Guidelines (2015).

Poor controlled Diabetes Mellites:

In our study, most of the patients were complicated with several systemic disease, and the average age was 73.42 years-old. The American Diabetes Association(ADA) considered the goals for treating glycemia in patients with Diabetes in elder patients with multiple coexisting chronic illness was HbA1c less than 8\%. [21] Patients with history of DM and baseline $\mathrm{HbA} 1 \mathrm{C}$ in 3 months more than $8 \%$ was defined poor controlled DM in our study. 


\section{Assessment}

Liver function: [22]

Liver function was assessed using the model for end-stage liver disease (MELD) score, Child-Pugh score, and albumin-bilirubin (ALBI) grade. The MELD score was calculated by the following formula: MELD score $=(9.57 \times$ loge creatinine $[\mathrm{mg} / \mathrm{dl}])+(3.78 \times$ loge bilirubin $[\mathrm{mg} / \mathrm{dl}])+(11.20 \times$ loge PT-INR $)+6.43$ and then rounded to the nearest integer. The lower limits for serum creatinine, serum bilirubin, and the PT-INR were fixed at 1 . The upper limit for serum creatinine was set at $4 \mathrm{mg} / \mathrm{dl}$. The ChildTurcotte-Pugh score uses 5 clinical parameters and gives a score of 1-3 for each parameter, namely, serum bilirubin, albumin, prothrombin time, ascites, and HE. The ALBI score was calculated by the following formula: $A L B I$ score $=(\log 10$ bilirubin $[4 \mathrm{~mol} / \mathrm{l}] \times 0.66)+($ albumin $[\mathrm{g} / \mathrm{l}] \times-0.085)$. The MELD score incorporates serum bilirubin, creatinine, and the prothrombin time-international normalized ratio (PTINR).

Renal function:

The renal function is estimated by Modification of Diet in Renal Disease (MDRD) equation: $186 \times \mathrm{Cr}^{-1.154} \times$ age $^{-0.203} \times(0.742$, if female) $\times(1.212$, if African American) 


\section{Results:}

Patient characteristics

A total 158 Taiwanese patients with HCV infection and CKD stage 3 were treated with interferon free DAA regimens. 28 patients were excluded due to loss follow or death during treating course $(n=14)$, incomplete baseline or SVR 12 data $(n=10)$, and those who had failed in HCV treatment $(n=4)$. The remained 130 patients were enrolled and analyzed in the study. (Figure1)

The overall eGFR for all patients had improvement at 12 weeks after end of treatment with DAA. $(p=0.025)$ (Figure $2 a)$ It increased from $49.071 \mathrm{ml} / \mathrm{min} / 1.73 \mathrm{~m}^{2}$ (95\% Cl 47.626-50.517) to $51.906 \mathrm{ml} / \mathrm{min} / 1.73 \mathrm{~m}^{2}$. (95\% Cl 48.475-53.338) The eGFR collected from patients remained in the study at SVR 96wks was 47.497 $\mathrm{ml} / \mathrm{min} / 1.73 \mathrm{~m}^{2}(95 \% \mathrm{Cl} 43.695-50.698)$, which was less than the eGFR at baseline $(p=0.310)$.

\section{Factors at baseline to renal function at SVR12}

According to the eGFR at Sustained Viral Response 12 weeks (SVR12), the patients could be divided into no-renal function declined group and renal function declined group by eGFR (Figure 2b). The change of eGFR at SVR12 weeks (Figure 2c) provide as a good predictor for the eGFR change at SVR96 weeks (Odd ratio 3.088, p $=0.0531)$. The baseline characteristics are presented in Table1. The mean age for the 
no-renal function declined group (72 years old) was younger than renal function declined group (75 years old) $(p=0.109)$. Female patients presented more percentage in the renal function declined group. $(p=0.157)$. Patients with Diabetes mellites were more in the renal function declined group with significance $(p=0.016)$. The odd ratio for DM was 2.6 (95\% Cl 1.25-5.405). (Table2) The other less significant factors to eGFR decline were the lower baseline MELD score $(p=0.072)$, history of malignancy $(p=0.076)$, and hypertension $(p=0.116)$ with or without $A C I / A R B$ use $(p=0.103)$. Initial CKD stage (3A or $3 B$ ) showed no significant difference $(p=0.548)$. The HCV genotypes among the patients were $1 a, 1 b, 2$, and 6 . The distribution of HCV genotypes within the groups was identical $(p=0.595)$. 


\section{Factors on DAA treatment to renal function at SVR12}

The serum creatine level, liver function, and HCV viral titer were collected at the

scheduled following time. The creatinine level increase $>0.3 \mathrm{mg} / \mathrm{dL}$ during the treating course was related to the eGFR decrease at SVR12 $(p=0.000)$. (Table 3) 2 patients developed infection related acute kidney injury, 1 patient had liver decompensation related renal function impairment, and 1 patient had developed Systemic lupus nephritis. The causes for other 22 of the 26 patients who had serum creatinine level increase had no confirmed etiology. The patients had serum creatinine level elevation during the treating course were found lower baseline HCV RNA titer $(p=0.024)$ and higher baseline MELD score $(p=0.008)$. Male patients had lower risk to get serum creatine increase as compared with female patients. $(p=0.059)$. Also, active malignant disease under concurrent treatment, either by Radiofrequency ablation, trans-arterial chemoembolization, immunotherapy, or systemic treatment, was related to the serum creatinine increase. $(p=0.044)$ Sofosbuvir-based regimen was not significantly associated with the risk of serum creatinine level increase. $(p=0.482)$ Integration of the characteristics with higher correlation to creatinine increase ( $p$ value $<0.1$ ) into a linear mixed effect model showed that the slopes of male sex was 0.109 ( $95 \% \mathrm{Cl}-0.070$ to $0.236 ; p=0.286)$, history of hypertension was $0.150(95 \% \mathrm{Cl}-0.019$ to $0.268 ; \mathrm{p}=0.088)$, history of 
cirrhosis was $0.064(95 \% \mathrm{Cl}-0.092$ to $0.189 ; \mathrm{p}=0.496)$, baseline MELD score was 0.067

(95\% $\mathrm{Cl}-0.027$ to $0.049 ; p=0.575)$, baseline CKD stage IIIA versus IIIB was -0.158

(95\% $\mathrm{Cl}-0.312$ to $0.045 ; \mathrm{p}=0.141)$, active malignancy was $0.148(95 \% \mathrm{Cl}-0.051$ to

$0.322 ; p=0.152)$, with concurrent malignancy treatment was $0.138(95 \% \mathrm{Cl}-0.087$ to

0.516; $p=0.162$ ) (Table.4) 
Discussion:

From a retrospective cohort study in the United States[23], top five variables associated with CKD in the chronically infected HCV patient were identified. These variables were: ACEIs, ARBs, congestive heart failure, hypertension, and Transplantation. In our study, DM with or without poor control is the most significant risk factor that was correlated to eGFR decline to the CKD stage 3 patients. Female sex, lower baseline MELD score, and hypertension with ACEI/ARB use showed a modest association with the eGFR decrease after achieving SVR.

Cirrhosis was reported to be a risk factor for AKI upon HCV treatment in several studies $[1,24]$. In ERCHIVES cohort study, patients with baseline cirrhosis had higher risk of eGFR decrease $>30 \mathrm{ml} / \mathrm{min} / 1.73 \mathrm{~m}^{2}$ at SVR12. [4] For cirrhosis and its effects on liver function, fib-4 score, MELD score, Child-Turcotte-Pugh (CTP) score and ALBI (Albumin-Bilirubin) grade are available tools. Comparing the parameters to liver function, MELD score was most related to the eGFR change in our patients. Intriguingly, the higher baseline MELD score was found in the non-renal function declined group at SVR12, but higher MELD score at baseline was a risk to creatinine increase more than $0.3 \mathrm{mg} / \mathrm{dL}$ upon DAA treatment. MELD score 9.67635 can be the cut of value (ROC curve, AUC 0.681) for predicting the renal function impairment. From the Target cohort study, cirrhosis was not a solitary risk factor to worsening 
renal function, MELD score more than 10 points was used to predict renal safety. [16] The renal function deterioration was resorted to diuretics use in the study, but the diuretics use and MELD score were no significantly associated in our patients $(P=0.439)$.

From CRIC study, a Cohort study in the US analyzing the eGFR reduction in DM and DM nephropathy, it was documented that the eGFR decline rate in DM patients at CKD stage $3 \mathrm{~A}$ and $3 \mathrm{~B}$ were about -0.19 to $-1.38 \mathrm{ml} / \mathrm{min} / 1.73 \mathrm{~m}^{2}$ per year and -1.38 to $-5.25 \mathrm{ml} / \mathrm{min} / 1.73 \mathrm{~m}^{2}$ per year, respectively. [25] The status of albuminuria played a role in the CKD progression. $\left(-1.75\right.$ and $-0.6 \mathrm{ml} / \mathrm{min} / 1.73 \mathrm{~m}^{2} \mathrm{per}$ year for albuminuric CKD and nonalbuminuric CKD) [26] In our study, although the albuminuria status of patients with DM history was not routinely measured at baseline, the average eGFR improved in DM patients with CKD stage 3 after a successful HCV elimination (average eGFR $47.30 \mathrm{ml} / \mathrm{min} / 1.73 \mathrm{~m}^{2}$ at base line and $47.28 \mathrm{ml} / \mathrm{min} / 1.73 \mathrm{~m}^{2}$ at SVR12, $\left.\mathrm{p}=0.208\right)$. This means, despite having comorbidity with DM, patients could still benefit from a successful HCV eradication to their eGFR. A link between HCV infection and type 2 DM has been proposed, and HCV infection was suggested to be related to the progression of diabetic nephropathy[10]. The DM nephropathy related CKD progression and its link to HCV clearance requires more study to our observation. 
Serum creatinine level increase more than $0.3 \mathrm{mg} / \mathrm{dL}$ in 48 hours is defined as acute kidney injury(AKI) in the KDIGO guideline [27]. Unusual increase of serum creatinine level is a sign to kidney injury. In our study, the renal function impairment during DAA treatment was highly associated with long-term eGFR decline after SVR. A retrospective observational cohort study in Boston [15] reported that AKI events were more common in patients with initial eGFR $<60 \mathrm{ml} / \mathrm{min} / 1.73 \mathrm{~m}^{2}$, and the injury could be irreversible even after discontinuation of the treatment. Some studies reported that the use of DAA may resulted in acute tubular necrosis (ATN) and acute interstitial nephritis (AIN). [24] A small case series at Miami reported that the renal biopsies at SVR22 to SVR 93 for patients treated with DAA who developed unknown cause of kidney injury had AIN, and three of the eight patients had worsening CKD and progressed to kidney failure requiring hemodialysis despite high dose steroid treatment. [28] Pathological data was not obtained from our patients, but male sex, cirrhosis with higher MELD score, concurrent malignancy treatment, lower baseline eGFR (CKD3B), and hypertension with or without ACEI/ARB use could potentially attribute the risk to renal function injury that could be possibly caused by AIN/ATN. Despite AKI incidence was reported low and reversible in most of the cases in clinical trials, the real-world data in our study offered a different idea. Renal function should be monitored more carefully. 
From Reveal-HCV study [29], chronic HCV infection with higher HCV viral load had higher CKD progression and development of ESRD. However, it was not discussed that if there is an association between the status of HCV viremia and renal function change. In our study, patients with lower baseline HCV viral titer were less likely to develop eGFR decline at SVR12, but there was a trend of lower risk of serum creatinine level increase for patients with higher HCV viral load. The reasons and mechanisms for the finding had not been discussed yet. The sample size was small in our study, and it might not be adequate to define a cut-off point value.

Our study has several limitations. For one thing, the included patients were sourced from a single healthcare system. Hence, it raised concerns about the generalizability to other populations. Also, many of the patients were incidentally found HCV infection in hepatocellular carcinoma survey, and the cancer related treatment might affect eGFR change. For another, a major limitation is the retrospective nature of the data collection. It is possible that patients developed AKI events were managed at hospitals outside our healthcare network and that the rate of AKI events was underestimated. Besides, it was difficult to trace all of the possible interfering factors, such as Chinese herb or healthy food use during HCV treatment. Furthermore, a survivorship bias for patients remained in our study at SVR96 may diminish the benefits on eGFR after a successful HCV clearance. 
In summary, the renal safety for patients in real world with moderate CKD on

DAA treatment till SVR96 can be assured as other published studies announced. [19,

30] Patients with DM history could have less benefit from HCV clearance by DAA

treatment. Active malignancy is not very associated with the eGFR decrease, but it

could be a risk to serum Creatinine level increase during the treatment course,

especially those who were treated with systemic treatments than local treatments.

Serum Creatinine level increase more than $0.3 \mathrm{mg} / \mathrm{dL}$ in treating course is a sign of

renal function deterioration, and which could be predisposed by male sex, lower

baseline renal function (CKD IIIB), hypertension, and cirrhosis with higher baseline

MELD score. 
List of abbreviations

CKD: chronic kidney disease; DAA: Direct-acting antiviral agents; SVR 12: sustained virologic response at 12 weeks; DM: Diabetes Mellites; eGFR: estimate Glomerular Filtration Rate; ESRD: End stage renal disease; HTN: hypertension; MELD: Model for End-Stage Liver Disease score; ALBI: Albumin-Bilirubin score; CTP: Child-TurcottePugh score. 


\section{Declarations:}

\section{Ethics approval and consent to participate}

The study was approved by VGHtpe Ethic Committee with principles of Declaration of Helsinki and the international conference on Harmonization for good clinical practice

\section{Consent for publication}

Written informed consent was obtained from the patients for publication

\section{Availability of data and materials}

The datasets used and analyzed during the current study are available from the corresponding author on reasonable request

\section{Competing interests}

The authors declare that there is no competing interest

\section{Funding}

This research did not receive any grant, funds, fees, and or support

\section{Authors' contributions}

Research idea and study design: Yen-Chi Hu; data acquisition: Chia-ling Lu,

; data analysis/interpretation: Yen-Chi Hu; statistical analysis: Yen-Chi Hu; and supervision or mentorship: Yi-Hsiang Huang, Ming-Chih Hou, Keng-Hsin Lan. Each author contributed important intellectual content during manuscript drafting or revision and accepts accountability for the overall work having ensure that questions 
pertaining to the accuracy or integrity of the work were appropriately investigated and resolved. The authors read and approved the final manuscript

\section{Acknowledgements}

Not applicable

\section{Authors' information}

1. Taipei Veterans General Hospital The Division of Gastroenterology \& Hepatology 


\section{References:}

1. Sara Yee Tartof, e.a., Kidney Function Decline in Patients with CKD and Untreated Hepatitis C Infection, in Clinical Journal of the American Society of Nephrology. 2018. p. 13: 1471-1478.

2. Khan, M.U., M.I. Mahmoud, and A.A. Butt, Hepatitis c virus and chronic kidney disease. Expert Rev Gastroenterol Hepatol, 2020. 14(7): p. 579-590.

3. Chen, Y.C., et al., $A$ nationwide cohort study suggests that hepatitis $C$ virus infection is associated with increased risk of chronic kidney disease. Kidney Int, 2014. 85(5): p. 1200-7.

4. Butt, A.A., et al., Do directly acting antiviral agents for HCV increase the risk of hepatic decompensation and decline in renal function? Results from ERCHIVES. Aliment Pharmacol Ther, 2017. 45(1): p. 150-159.

5. Kao, C.C., et al., Association of Renal Function and Direct-Acting Antiviral Agents for HCV: A Network Meta-Analysis. J Clin Med, 2018. 7(10).

6. Carrier, P., Anti-hepatitis C virus drugs and kidney, in World journal of Hepatology. 2016. p. 1343-1353.

7. David Roth, e.a., KDOQI US Commentary on the 2018 KDIGO Clinical, in American journal of kidney disease. 2020. p. 665-683.

8. Stanislas, e.a., Hepatitis C virus and the kidney, in Nature reviews Nephrology. 2019.

9. Haesuk Park, e.a., Chronic Hepatitis C Virus (HCV) Increases the Risk of Chronic Kidney Disease (CKD) While Effective HCV Treatment Decreases the Incidence of CKD, in Hepatology. 2018. p. 492-504.

10. Ozkok, A. and A. Yildiz, Hepatitis C virus associated glomerulopathies. World J Gastroenterol, 2014. 20(24): p. 7544-54.

11. Driedger, M., C. Galanakis, and C. Cooper, Direct acting antiviral HCV treatment does not influence renal function. Medicine (Baltimore), 2020. 99(22): p. e20436.

12. Hsu, Y.C., et al., Association between antiviral treatment and extrahepatic outcomes in patients with hepatitis C virus infection. Gut, 2015. 64(3): p. 495503.

13. Feng, B., et al., Effect of interferon-alpha-based antiviral therapy on hepatitis $C$ virus-associated glomerulonephritis: a meta-analysis. Nephrol Dial Transplant, 2012. 27(2): p. 640-6.

14. D'Ambrosio, R., et al., Renal safety in 3264 HCV patients treated with DAA- 
based regimens: Results from a large Italian real-life study. Dig Liver Dis, 2020. 52(2): p. 190-198.

15. Sise, M.E., et al., Direct-acting antiviral therapy slows kidney function decline in patients with Hepatitis C virus infection and chronic kidney disease. Kidney Int, 2020. 97(1): p. 193-201.

16. Saxena, V., et al., Safety and efficacy of sofosbuvir-containing regimens in hepatitis C-infected patients with impaired renal function. Liver Int, 2016. 36(6): p. 807-16.

17. Foucher, J., et al., Diagnosis of cirrhosis by transient elastography (FibroScan): a prospective study. Gut, 2006. 55(3): p. 403-8.

18. Gordon, S.C., et al., Safety profile of boceprevir and telaprevir in chronic hepatitis C: real world experience from HCV-TARGET. J Hepatol, 2015. 62(2): p. 286-93.

19. Sise, M.E., et al., Effect of Sofosbuvir-Based Hepatitis C Virus Therapy on Kidney Function in Patients with CKD. Clin J Am Soc Nephrol, 2017. 12(10): p. 1615-1623.

20. European Association for the Study of the Liver. Electronic address, e.e.e. and L. European Association for the Study of the, EASL Clinical Practice Guidelines for the management of patients with decompensated cirrhosis. J Hepatol, 2018. 69(2): p. 406-460.

21. Professional Practice Committee: Standards of Medical Care in Diabetes-2021. Diabetes Care, 2021. 44(Suppl 1): p. S3.

22. Maesaka, K., et al., Clinical course of hepatitis $C$ virus-positive patients with decompensated liver cirrhosis in the era of direct-acting antiviral treatment. Hepatol Res, 2021.

23. Park, H., Chronic Hepatitis C Virus (HCV) Increases the Risk of Chronic Kidney Disease (CKD) While Effective HCV Treatment Decreases the Incidence of CKD, in Hepatology. 2018. p. VOL. 67, NO. 2.

24. Brown, P.R., et al., Acute Kidney Injury in Patients Undergoing Chronic Hepatitis C Virus Treatment With Ledipasvir/Sofosbuvir. Hepatol Commun, 2018. 2(10): p. 1172-1178.

25. Koye, D.N., et al., Risk of Progression of Nonalbuminuric CKD to End-Stage Kidney Disease in People With Diabetes: The CRIC (Chronic Renal Insufficiency Cohort) Study. Am J Kidney Dis, 2018. 72(5): p. 653-661.

26. Buyadaa, O., et al., Risk of Rapid Kidney Function Decline, All-Cause Mortality, and Major Cardiovascular Events in Nonalbuminuric Chronic Kidney Disease in Type 2 Diabetes. Diabetes Care, 2020. 43(1): p. 122-129.

27. Uhlig, K., KDIGO Clinical Practice Guideline for Acute Kidney Injury. Kidney 
International Supplements, 2012. 2(1).

28. Duque, J.C., et al., Acute interstitial nephritis following treatment with directacting antiviral agents in hepatitis $C$ virus-infected patients: $A$ case series. Clin Nephrol, 2021. 95(1): p. 22-27.

29. Lai, T.S., et al., Hepatitis C viral load, genotype, and increased risk of developing end-stage renal disease: REVEAL-HCV study. Hepatology, 2017. 66(3): p. 784-793.

30. Medeiros, T., et al., Renal safety after one year of sofosbuvir-based therapy for chronic hepatitis C: A Brazilian "real-life" study. J Clin Pharm Ther, 2018. 43(5): p. 707-713. 
Figures

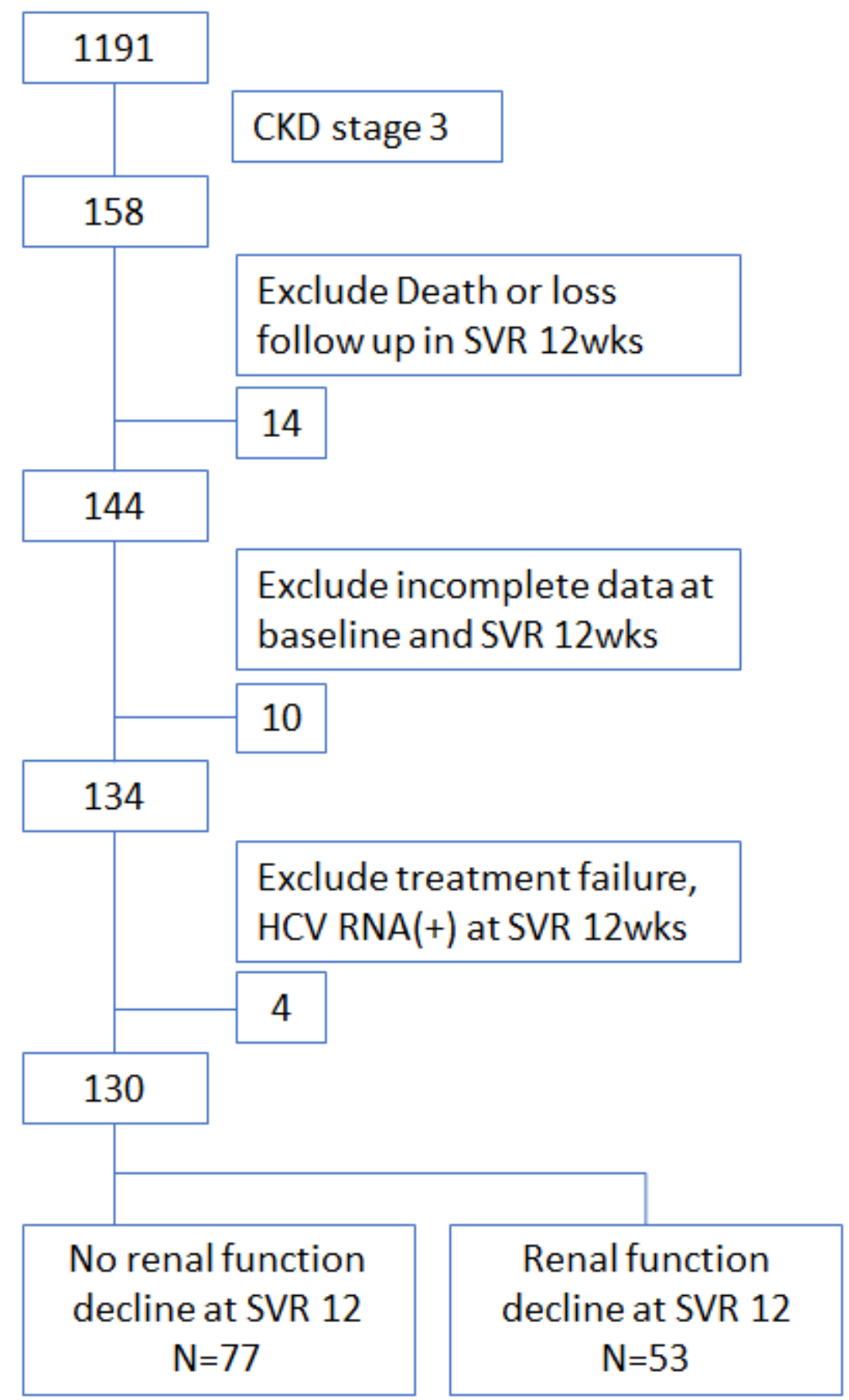

Figure 1

study flow 

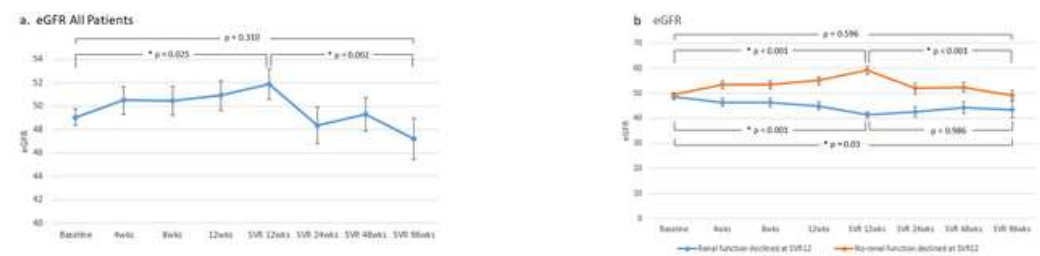

Figure $2 c$

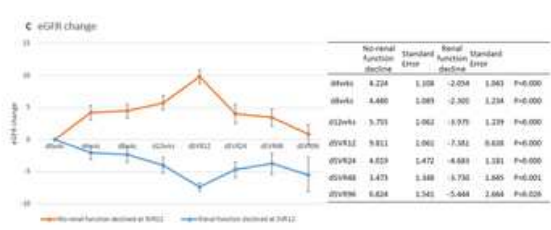

Figure 2

Average estimated glomerular filtration rate (eGFR) at baseline, 4wks, 8wks, 12wks, SVR12, SVR24, SVR48, and SVR96 a. The eGFR trend from baseline to SVR 12wks, all patients. b. The eGFR trend from baseline to SVR $96 \mathrm{wks}$ in no renal function declined group and renal function declined group at SVR12wks. The data after SVR12wks was calculated from the data of the non-loss follow up patients. The best eGFR improvement in the non-declined group was at SVR 12wks, and the eGFR decreased. At SVR $96 \mathrm{wks}$, the eGFR was modest $\mathrm{c}$. The trend of eGFR change compared with baseline data from baseline to SVR 96weeks. The data after SVR12wks was calculated from the data of the non-loss follow up patients. SVR: sustain viral response, wks: weeks

\section{Supplementary Files}

This is a list of supplementary files associated with this preprint. Click to download.

- Table1.pptx

- Table2.pptx

- Table3.pptx

- Table4.pptx 